\title{
Radiation effects on two-dimensional materials
}

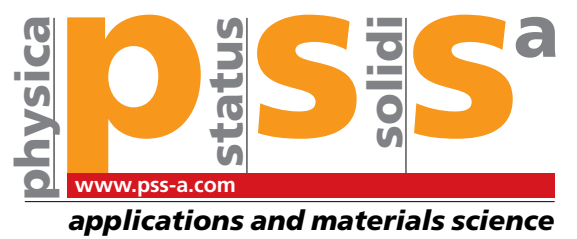

Feature Article

\author{
R. C. Walker II ${ }^{1,2}$, T. Shi ${ }^{3,4}$, E. C. Silva ${ }^{5}$, I. Jovanovic ${ }^{4}$, and J. A. Robinson ${ }^{*, 1,2}$ \\ ${ }^{1}$ Department of Materials Science, Penn State, University Park, PA 16802, USA \\ ${ }^{2}$ Center for Two-Dimensional Layered Materials, Penn State, University Park, PA 16802, USA \\ ${ }^{3}$ Department of Mechanical and Nuclear Engineering, Penn State, University Park, PA 16802, USA \\ ${ }^{4}$ Department of Nuclear Engineering and Radiological Sciences, University of Michigan, Ann Arbor, MI 48109, USA \\ ${ }^{5}$ GlobalFoundries, Malta, NY 12020, USA
}

Received 6 June 2016, revised 28 August 2016, accepted 30 August 2016

Published online 26 September 2016

Keywords graphene, radiation materials science, single event effect, transition metal dichalcogenides, two-dimensional materials

*Corresponding author: e-mail jrobinson@psu.edu, Phone: 8148638567

The effects of electromagnetic and particle irradiation on twodimensional materials (2DMs) are discussed in this review. Radiation creates defects that impact the structure and electronic performance of materials. Determining the impact of these defects is important for developing 2DM-based devices for use in high-radiation environments, such as space or nuclear reactors. As such, most experimental studies have been focused on determining total ionizing dose damage to 2DMs and devices. Total dose experiments using X-rays, gamma rays, electrons, protons, and heavy ions are summarized in this review. We briefly discuss the possibility of investigating single event effects in 2DMs based on initial ion beam irradiation experiments and the development of 2DM-based integrated circuits. Additionally, beneficial uses of irradiation such as ion implantation to dope materials or electron-beam and helium-beam etching to shape materials have begun to be used on 2DMs and are reviewed as well. For non-ionizing radiation, such as low-energy photons, we review the literature on 2DM-based photo-detection from terahertz to UV. The majority of photo-detecting devices operate in the visible and UV range, and for this reason they are the focus of this review. However, we review the progress in developing 2DMs for detecting infrared and terahertz radiation.
1 Introduction The isolation of graphene layers in 2004 [1] marked the start of a new area of research in twodimensional materials (2DMs). The broad goals of this research thrust include understanding basic properties that would enable many applications (transistors, catalysts, sensors, dielectrics, etc.) and uncovering new physics from a wide range of candidate materials (e.g., graphene, transition metal dichalcogenides (TMDs), hexagonal boron nitride (hBN), etc.). For example, graphene is known to have excellent electron mobility due to its massless charge carriers, but it lacks a band gap and thus distinct ON and OFF states. Semiconducting materials, including several transition metal dichalcogenides (TMDs) show a reduced mobility with significant improvements in their ON/OFF ratio. Hexagonal boron nitride $(\mathrm{hBN})$ is an insulator with a highly stable graphitic structure that has been used as a high temperature ceramic. These atomically thin materials have a high sensitivity to extrinsic effects [2], such doping from adsorbed gaseous species and carrier scattering at substrate and dielectric interface traps. In this review, we summarize the influence of another aspect of the environment: incident ionizing radiation in the form of photons, neutrons or charged particles. First, a general description of the types and impacts of defects on electronic properties is presented. The effects of low energy photons - i.e. terahertz radiation, infrared (IR) radiation, visible light, and ultraviolet (UV) light - are subsequently discussed. Irradiation from highenergy photons (X-rays and gamma rays) is then succinctly discussed. Finally, bombardment by charged particles is discussed.

2 Defect types and impacts on the electronic properties of 2DMs It is well-known that the inclusion of defects inside a semiconductor's lattice can alter - and usually degrade - device performance (see Table 1 for a summary). This remains true for irradiation-induced 
Table 1 Summary of the types, causes, and effects of defect states in 2DMs created due to radiation exposure.

\begin{tabular}{|c|c|c|c|}
\hline defect & particle & impact & reference \\
\hline generation/recombination site & heavy charged particles & $\begin{array}{l}\text { scattering; carrier } \\
\text { concentration modulation }\end{array}$ & {$[3]$} \\
\hline trap state & heavy charged particles & $\begin{array}{l}\text { scattering site; carrier lifetime } \\
\text { reduction; charge build-up }\end{array}$ & {$[3-7]$} \\
\hline dopant & $\begin{array}{l}\text { heavy charged particles, } \\
\text { ion beam }\end{array}$ & scattering site; charge build-up & {$[3,8]$} \\
\hline tunneling defect & heavy charged particles & leakage current & [3] \\
\hline vacancy & $\begin{array}{l}\text { electrons, gamma rays, } \\
\text { ion beam, X-rays }\end{array}$ & $\begin{array}{l}\text { deep level traps and direct to } \\
\text { indirect band-gap transition } \\
\text { in } \mathrm{WSe}_{2} \text {; scattering sites in } \\
\text { graphene; deep acceptor or } \\
\text { shallow donor in } \mathrm{MoS}_{2}\end{array}$ & [9-17], this work \\
\hline interface states & $\begin{array}{l}\text { gamma rays, heavy charged } \\
\text { particles }\end{array}$ & $\begin{array}{l}\text { scattering site; carrier lifetime } \\
\text { reduction, carrier concentration } \\
\text { modulation }\end{array}$ & {$[6,18-20]$} \\
\hline
\end{tabular}

defects. For example, irradiation from cosmic rays is a significant concern for space applications because of their destructive power [3]. However, 2DMs such as graphene exhibit reduced probabilities of interaction between ions and the 2D layers $[9,10]$, and so the damage from high energy ions occurs primarily in the substrate, creating various defects near the substrate and 2DM interface such as generation/recombination sites, traps, compensators, and tunneling sites [3]. Each of these defects has a distinct impact on device performance. Generation sites are of major concern in semi-insulating materials, while recombination sites are of more concern in heavily doped materials. These types of sites have the most impact if they lie near the middle of the band gap. Traps degrade the performance of charge coupled devices, while compensators degrade the performance of bipolar junction transistors. Tunneling defects can lead to unacceptable leakage currents.

These defects originate from the ionization and displacement of atoms energized by incident radiation. For example, ionized silicon dioxide can trap holes at interfaces via the migration and build-up of both holes and traps, causing major shifts in the threshold voltage. Current anneals can be used to repair the damage in radiationhardened oxides, depending on the electric field applied and the thickness of the gate oxide layer. A sufficiently large number of trapped holes (i.e., 50\% of holes generated are trapped instead of collected) cannot be fully repaired by annealing [4, 5]. A larger percentage of trapped holes also increases the amount of time needed for the oxide to heal, which can make it appear to be permanently damaged. It has been estimated that for silicon dioxide, radiation hardness can be achieved if the percentage of trapped holes can be kept below a threshold of $10 \%$. Photodetectors and field effect transistors (FETs) based on silicon commonly fail in this manner after long periods of operation [3]. Note that FETs can also fail from mobility degradation in the long term. Substrate defects such as these can damage devices either by accumulation of smaller interactions or in a single event. Single-particle events may cause permanent failures in the specific cases of memory and power devices [3]; more details are given in Section 7. They can also cause latchup (short circuit) in silicon-based FETs. As silicon is likely to be used as a substrate material for devices incorporating $2 \mathrm{D}$ materials, these damage and failure modes will remain relevant into the future. They may also be used as a guide for the future when selecting other substrates, and maintaining the goal of minimizing the number of defects generated by radiation.

Substrate defects influence 2DMs at interfaces. However, other defects can be generated inside the channel and directly influence its electronic properties. For example, chalcogenide vacancies are preferentially generated upon the irradiation of TMDs with a lighter chalcogenide and heavier transition metal [21]. The effect of these vacancies on the electronic properties of $\mathrm{MoS}_{2}$ was documented in a thorough first-principles study of various vacancy types [16]. They appear to create deep acceptor states within the $\mathrm{MoS}_{2}$ band-gap. However, reducing the sulfur content of $\mathrm{MoS}_{2}$ has also been shown to increase the mobility, ON/OFF ratio and n-type behavior of devices [12]. This effect was also attributed to the sulfur vacancy, which was claimed to act as a shallow donor [12]. Meanwhile, a combined experimental and theoretical study of charge transport in $\mathrm{MoS}_{2}$ has revealed that the sulfur vacancy should act as a deep donor [17]. As such, the true influence of the sulfur vacancy is still to be determined and requires further investigation. We have modeled the effect of selenium vacancy generation on the electronic properties of $\mathrm{WSe}_{2}$ by calculating the influence of chalcogenide single and double vacancies on the band structure of $\mathrm{WSe}_{2}$ using density functional theory via the VASP simulation package [22] (see Fig. 1). These structural defects were considered due to the expected preferential sputtering of chalcogen atoms. Vacancies were generated in the simulation by manually removing selenium 


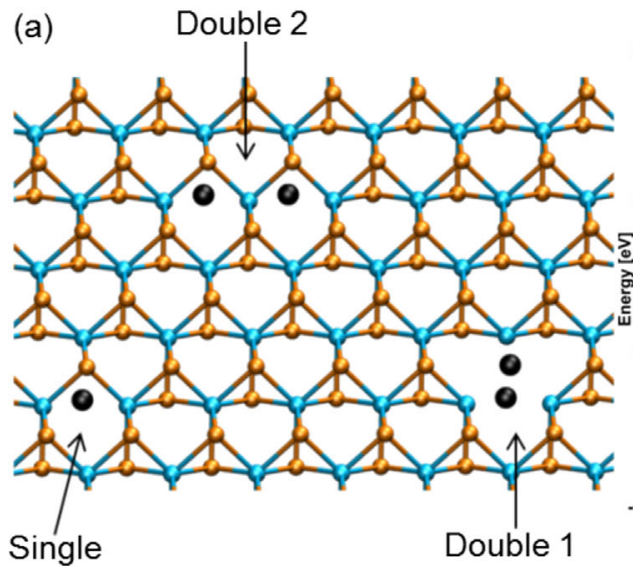

(b) Pristine

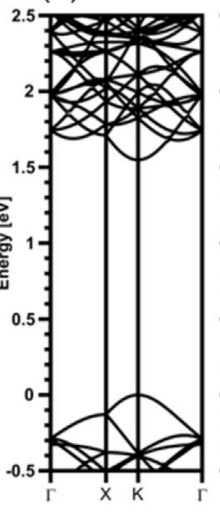

(c) Single

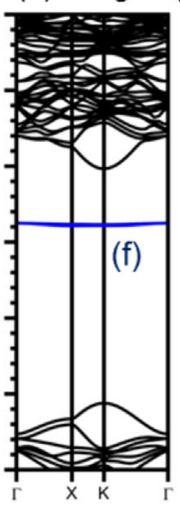

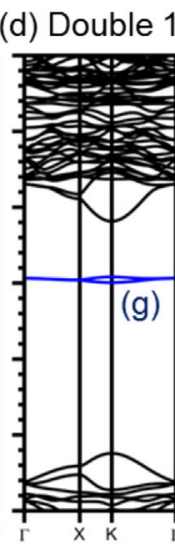

(e) Double 2

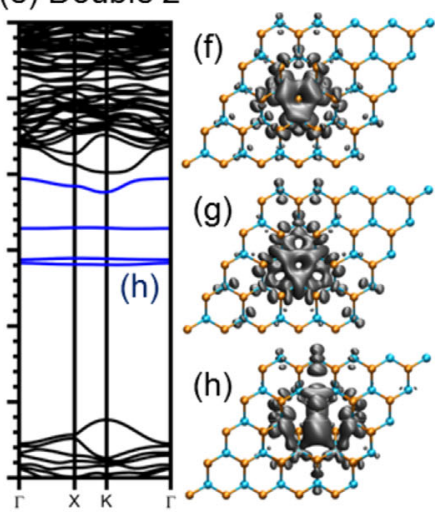

Figure 1 (a) $\mathrm{WSe}_{2}$ (W in blue; Se in orange) with a single chalcogenide (single; Se) vacancy, an $a-b$ axis double (double 2; 2Se) vacancy and a $c$-axis double (double 1; $\mathrm{Se}_{2}$ ) vacancy. Dark spheres are missing atoms. (b) Band structure for pristine WSe $\mathrm{C}_{2}$, while (c)-(e) show the same for $\mathrm{WSe}_{2}$ with a vacancy of each type. Mid-gap states are apparent after vacancy formation, and a direct to indirect band gap transition occurs after the formation of 2 Se vacancies. Linear density of state calculations corresponding to (c)-(e) are shown in (f)-(h), and show the loss of electronic symmetry resulting from the 2 Se vacancy.

atoms from a $5 \times 5$ supercell and then performing a full structural optimization until all forces were below the $0.01 \mathrm{eV}^{-1}$ threshold. Each vacancy creates localized carrier-trapping deep states within the band gap (see Fig. 1b-d). The removal of a single chalcogenide (Se) or two along the $c$-axis $\left(\mathrm{Se}_{2}\right)$ preserves the direct band gap of monolayer $\mathrm{WSe}_{2}$. However, the removal of two adjacent selenium atoms in the same chalcogenide layer (2Se) causes a transition from a direct to an indirect band gap, which was correlated to the change in the local crystal structure (see Fig. 1e-g).

3 Photon irradiation Responses to incident electromagnetic radiation can be characterized by several metrics: responsivity $(\mathrm{A} / \mathrm{W})$, quantum efficiency $(\%)$, detectivity (Jones, or $\mathrm{cm}-\mathrm{Hz}^{1 / 2} / \mathrm{W}$ ), and noise equivalent power $\left(\mathrm{W} / \mathrm{Hz}^{1 / 2}\right)$. Responsivity $(\mathrm{R})$ is the primary metric used in the literature and by industry, and is the main metric utilized in this review. It is defined as the photocurrent per unit power of the incident light, and can be measured by shining light of fixed wavelength onto back-gated FETs and recording changes in the device current. The responsivity is controlled not only by the material properties of the detector, but also by the processing methods and device architecture, as will be apparent in the following subsections.

3.1 Attenuation The penetrative power of incident radiation is controlled by three factors: particle type, particle energy, and the attenuation occurring inside the sample. In general, photon flux through solids decreases exponentially through the thickness. Due to the atomically thin nature of 2DMs, most incident radiation passes through without interaction. Graphene absorbs about $2.3 \%$ of incident infrared and visible light due to its reduced dimensionality and metallic nature [23], which is small in absolute terms but large for an atomically thick layer. Several varieties of molybdenum- and tungsten-based TMDs also exhibit large absorbance (5-10\%) as monolayers [24]. Graphene, with its lack of a band-gap, does not display any preferential absorption of photon energies, which makes it useful for photo-detection across a large energy range, as will be clear in the following sections.

3.2 Terahertz Devices for the detection of terahertz radiation are a newly emerging area of research. Terahertz waves lie in the region of the electromagnetic spectrum between radio waves and infrared, and have historically evaded significant technological usage. This is because conventional microwave and optical technologies lose their effectiveness within this frequency range $(0.3-3 \mathrm{THz})$. Bridging this so-called "terahertz gap" is an active area of research due to predicted applications for terahertz detection such as spectroscopic identification of hazardous substances, detecting dielectric materials, and fast imaging [25].

Terahertz detection typically occurs in FETs through the excitation and resonance of plasma waves in the transistor channel, as these particles are too low in energy to excite electrons across a band gap [26]. To maximize this resonance, devices with very high mobility are necessary, which has led to the development of graphene terahertz detectors [25, 27]. The source and top-gate of these FETs are used as amplifiers to convert the photocurrent into a voltage signal between the source and drain. To take advantage of this change in voltage, an asymmetric design is needed to induce an asymmetric photocurrent in the symmetric graphene layers [28]. Broadband detection is the usual mode for these devices. However, quantized detection is possible as the channel length increases and the terahertz wave energy increases. A maximum 50\% broadband 
absorption of incident radiation is possible by manipulating the conductivity of graphene, due to coupling between the electrical and optical conductivities of graphene. At the minimum conductance, the absorption remains below $5 \%$, comparable to general UV-vis light absorption in TMDs [23]. Preliminary devices exhibited a voltage responsivity of $0.1 \mathrm{~V} / \mathrm{W}[25,28]$. This was argued to be a lower limit due to inefficiencies in funneling incident light onto the channel. Improvements in device design or material properties would improve detector performance. In more recent results, a voltage responsivity of $1.2 \mathrm{~V} / \mathrm{W}$ ( or $1.3 \mathrm{~mA} / \mathrm{W}$ ) has been achieved with bilayer graphene [29], and a voltage responsivity of $715 \mathrm{~V} / \mathrm{W}$ to $119 \mu \mathrm{m}$ terahertz waves has been obtained in exfoliated graphene through the use of the photothermoelectric effect [30]. The first result is comparable to commercial $\mathrm{THz}$ detectors and the second result indicates that fast, high-response and low noise detectors are possible with graphene. However, the second result bears repeating with chemical vapor deposition (CVD) or epitaxially grown graphene in order to be proven practical.

3.3 Infrared The detection of infrared photons is necessary for applications using IR lasers, such as optical communication, or detection and imaging. The use of $2 \mathrm{D}$ materials in IR photodetectors has not been investigated as thoroughly as in UV and visible light detectors due to the reduced or non-existent responsivity to IR light from nongraphene materials. The mechanism for photon detection for graphene in the infrared regime is often direct absorption; individual layers of graphene can only absorb up to $2.3 \%$ incident light unaided [23]. Several methods have been proposed to increase this absorption. For example, a mirror-backed photonic crystal slab has been proposed to increase photon absorbance up to $100 \%$, and increases in absorbance from 2.3 to nearly $100 \%$ at a tailored resonance frequency have been demonstrated experimentally [31].

3.4 Visible and ultraviolet Investigating the detection of visible and UV light by semiconducting 2DMs has led to a proliferation ofphotodetectors with responsivity spanning approximately 15 orders of magnitude (see Fig. 2a-d) across a wide variety of materials systems, including graphene [32-38] and reduced graphene oxide (rGO) [39, 40]; black phosphorous [41-44]; III-VI layered compounds ( $\mathrm{GaX}$ [45-50], InX [51-54], $\mathrm{In}_{2} \mathrm{X}_{3}$ [55]); TMDs $\left(\mathrm{MoS}_{2}[41,56-65], \mathrm{ReS}_{2}[66], \mathrm{WX}_{2}[64,67-70]\right)$; and postTMDs ( $\mathrm{SnS}_{2}$ [71-73]); $\mathrm{Bi}_{2} \mathrm{Te}_{3}$ [74]. From this vast and everincreasing amount of data, there are several key points that emerge:

Graphene by itself is not an excellent photodetector. This is apparent from the performance of the graphenebased photodetectors [32-35, 38] demonstrated in literature (see Fig. 2a). The root causes of the poor responsivity of graphene are its metallic nature and limited light absorption.
Thus, increasing the responsivity of graphene in the visible and near-UV regions has been achieved by introducing a band gap, either by creating rGO [39, 40, 75] or quantum dot-like structures (GQDS [36, 37]). The full conceptual details of the GQDS are discussed elsewhere [36]; in short, introducing a band gap and electron trapping mid-gap states allows for carrier multiplication and recirculation. These two factors led to a three order of magnitude increase in responsivity via a tunable process.

For large responsivity, graphene should be used as a contact, not as a photo-active element. Several studies [53, $57,76-82]$ on the photodetecting potential of graphene heterojunctions have shown that careful selection of materials allows for significant enhancement of responsivity by up to $\sim 10$ orders of magnitude (see Fig. $2 b$ ). Highly sensitive devices with $R>10^{7}$ can be developed depending on the materials in the heterojunction. The mechanism for this improvement is described as "quantum carrier reinvestment," where carriers generated in the other material migrate to the graphene and recirculate many times before they recombine [77].

The choice of substrate is important. This can be observed not only through graphene heterojunctions, but also though studies of suspended graphene $[32,83]$ and photo-sensors based on gallium sulfide $(\mathrm{GaS})$ [45]. The close proximity of electrons and holes in 2DMs to the substrate aids trapping at any interfacial defects. Reducing interface scattering by using a less defective substrate increases the responsivity [45] (see Fig. 2c). One avenue to reduce scattering is by etching away excess substrate area, leaving behind freestanding channels. The removal of substrate-assisted carrier cooling allows for some improvement in the responsivity when compared to fully supported devices operating with the same mechanism $[32,83]$.

The sample thickness is important. Reducing the number of layers in layered materials leads to alterations in the band structure that usually increases the band gap. Manipulation of the thickness to add sensitivity of lower energy light has been demonstrated with $\mathrm{MoS}_{2}$-based devices. Monolayer $\mathrm{MoS}_{2}$ with a band gap of $1.83 \mathrm{eV}$ does not capture the full visible spectrum, but bilayer $\left(E_{\mathrm{g}}=1.65 \mathrm{eV}\right)$ and tri-layer $\left(E_{\mathrm{g}}=1.35 \mathrm{eV}\right) \mathrm{MoS}_{2}$ can respond to red [84] near-IR [60] light. Achieving this functionality in monolayer $\mathrm{MoS}_{2}$ requires another method of band gap modulation such as using dye-sensitization [61] or photothermoelectric effect [85]. However, stacking multiple layers leads to significant reduction in $R$, as shown by studies on layer-dependent responsivity for $\mathrm{MoS}_{2}$ [86] and GaSe [46].

The sample preparation process is important. For example, exfoliated gallium telluride (GaTe) layers can reach $R \sim 10^{4} \mathrm{~A} / \mathrm{W}$ [48], while GaTe layers grown via chemical vapor deposition have exhibited $R \sim 1 \mathrm{~A} / \mathrm{W}$ [49]. This significant degradation in performance was attributed to defects generated from the growth process, Ga vacancies in particular. It has been shown that suppressing these defects allows for high-performance phototransistors [87]. 

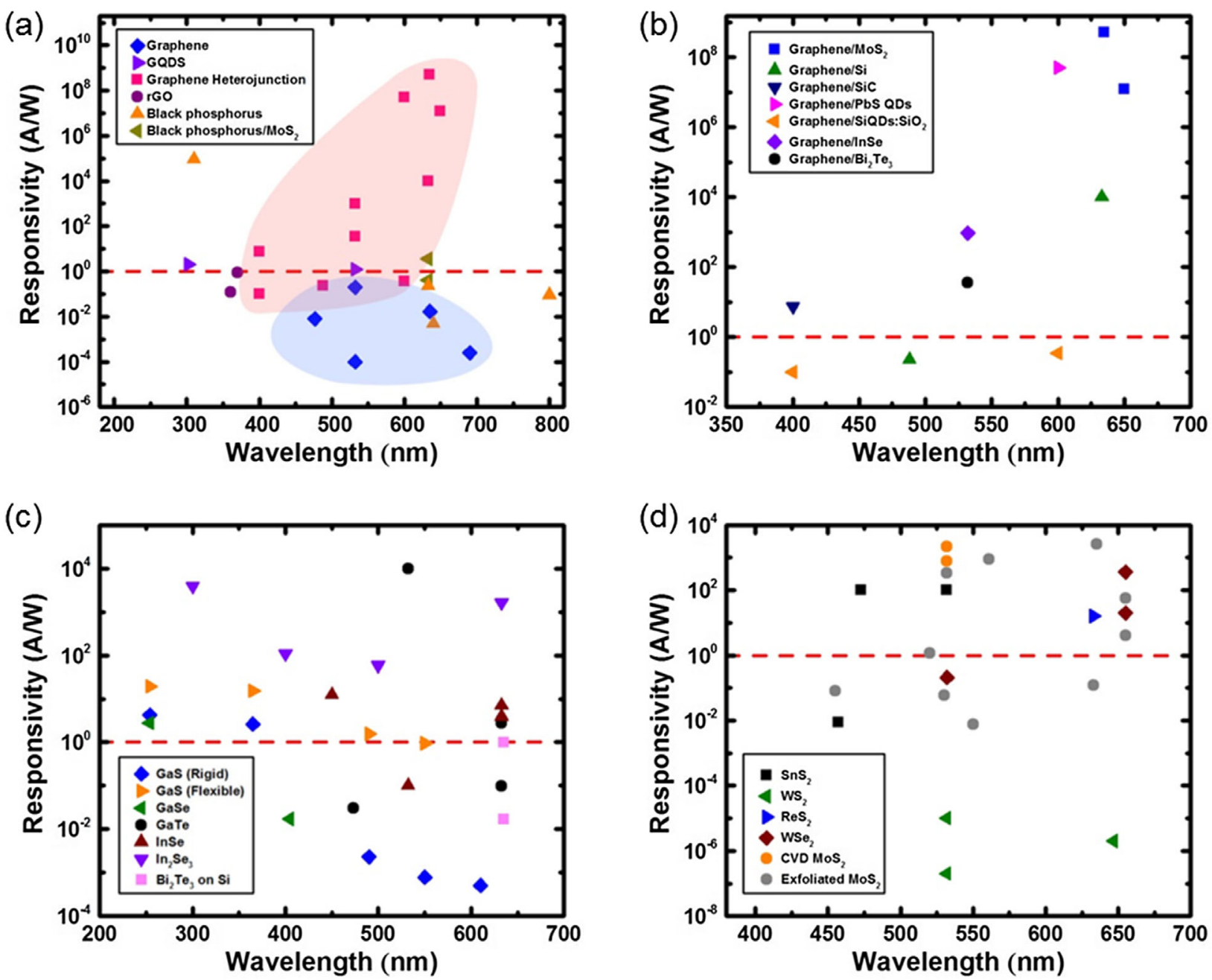

Figure 2 (a) Responsivity of graphene-like 2DMs to visible and UV light. The dashed line in all plots indicates the $R=1$ level that must be surpassed for practical applications [136]. (b) Responsivity of graphene heterojunctions to visible and UV light. (c) Responsivity of III-VI and V-VI 2DMs to visible and UV light. (d) Responsivity of TMDs to visible and UV light. Studies of these materials have primarily been focused in the visible range due to the corresponding TMD band gaps, leaving the UV-regime performance of these materials yet to be determined.

This idea can be extended to all 2DMs, and the critical defect species must be determined for each one. For example, in the case of a material such as rGO, the electrical [75] and optical [88] properties clearly can be controlled by manipulating the oxygen content. One preliminary study has also shown that using an appropriate surface dopant (p-type for $\mathrm{WSe}_{2}$ and n-type for $\mathrm{MoS}_{2}$ ) can be used to enhance photoresponsivity by over an order of magnitude [64]. However, the selection of an air-stable dopant has yet to be fully investigated.

$\mathrm{MoS}_{2}$ in particular has been heavily studied using exfoliated and as-grown materials. A comparison using this literature shows that the first attempts at making photodetectors with as-grown materials are comparable to the best devices made using exfoliated $\mathrm{MoS}_{2}$ (see Fig. 2d). These samples, grown by reacting molybdenum oxide with sulfur powder, exhibit a responsivity of $R \sim 1000 \mathrm{~A} / \mathrm{W}$ and are improved by vacuum anneals that remove adsorbed oxygen [56]. For comparison, achieving the same level of responsivity in exfoliated material requires either a special surface treatment (e.g., oxygen plasma cleaning [59] or a ferroelectric surface dopant [65]). Furthermore, exfoliated material cannot be used to support large-scale device fabrication, while the responsivity enhancers used for exfoliated $\mathrm{MoS}_{2}$ can be applied to as-grown material. Thus, to achieve wide-scale use of practical 2DM-based devices, we recommend that robust growth processes be pursued.

3.5 High-energy photons Gamma rays $(E>$ $\sim 100 \mathrm{keV})$ and X-rays $(\sim 100 \mathrm{keV}>E>\sim 100 \mathrm{eV})$ are high-energy photons released from nuclear reactions and decelerated electrons, respectively. In the context of semiconductors, gamma rays and X-rays can damage devices by creating vacancies or other ionized defects. 
Graphene is an excellent match for high energy photon detection, as it is highly sensitive to the local changes in its electric field caused by X-rays [89, 90] and gamma rays [91, 92]. These changes are detectable using FET devices by tracking changes in device performance as it is exposed to an ionizing radiation source. Detection of these radiation sources is important within the contexts of radiation protection, monitoring special nuclear material [89], radiation-based medical diagnostics and treatment [93], and in other areas of nuclear science and engineering. Gamma and X-ray detection in a graphene FET are measured as modulations of the Dirac voltage, or the voltage corresponding to minimized conductance in the graphene. This modulation occurs due to changes in the local electrical field caused by radiation-induced charged states $[89,90]$. X-ray exposure can also be used to reduce graphene oxide to make rGO, and greater reduction occurs when it is included within a heterostructure with hBN [92]. However, gamma rays can achieve similar levels of reduction while reducing the total dose by several orders of magnitude due to their higher energy.

Gamma rays generate energetic free electrons and positive ions in solids by mechanisms such as Compton scattering [92]. These free electrons then interact with nearby atoms to ionize and dislodge them, creating vacancies and interstitials [91, 94]. In graphene, this disorder is measurable via Raman spectroscopy by tracking changes in its characteristic peaks. These changes signify the onset of damage and disorder. Maximum structural disorder is generated at an intermediate doses due to the re-structuring that occurs at large doses via radiationassisted diffusion and annealing, and so the total disorder saturates to a reduced extent [94]. At low doses, gamma rays can be used to increase the carrier density with little defect generation in graphene FETs [91]. Damage can also be measured by oxidation in air, and more oxidation by various functional groups (i.e., hydroxyls, ethers, carboxyls, etc.) occurs as the dose increases [94]. However, gamma rays can also be used beneficially to produce rGO when combined with chemical reducing agents $[95,96]$.

4 Electron irradiation Electron damage to materials is important due to the two major electron-using characterization tools: the scanning electron microscope (SEM) and transmission electron microscope (TEM). A full treatment of the damage is given elsewhere [97]; in short, elastic and inelastic scattering events give rise to the following six damage mechanisms: displacement, sputtering, heating, charging, radiolysis, and radiation-induced contamination (see Fig. 3). Damage to 2DMs occurs mainly through sputtering and displacement for metals or radiolysis and charging for insulators and semiconductors $[13,14,21,94$, 98-101]. In order to sputter away an atom, the electron must be able to transfer enough energy to overcome the threshold for knock-on damage [13]; the determination of this energy for graphene has been done [102], and the (a)
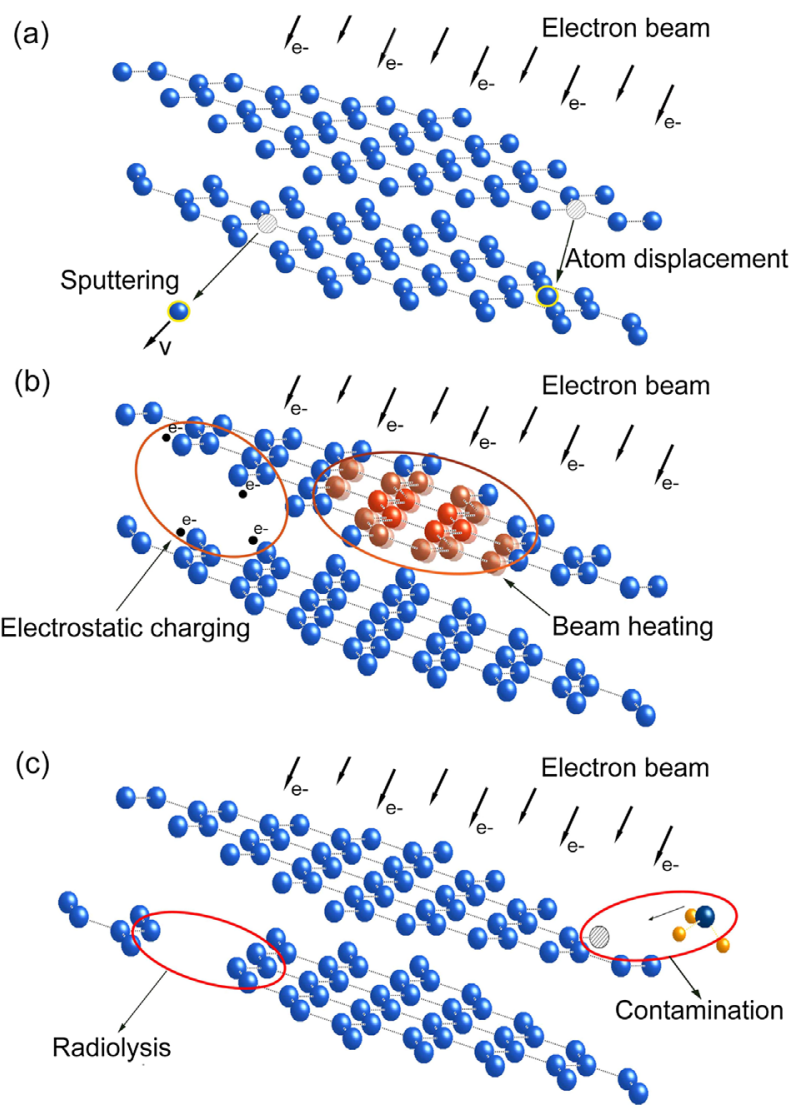

Figure 3 Illustration of electron-beam induced damage processes: (a) sputtering and atom displacement; (b) electrostatic charging and beam heating; (c) radiolysis and contamination.

same must be done for other 2DMs. For insulators, the charging induced by the electron-beam can also cause electrostatic repulsion and lead to the so-called local "Coulomb explosion" [13]; however, it has so far only been observed in hBN nanotubes [103].

Electron beam irradiation of graphene via TEM has been shown to generate stable defect structures such as pentagon-heptagon pairs (including the Stone-Wales defect, a rotation of a $\mathrm{C}-\mathrm{C}$ bond by $90^{\circ}$ ), adatoms, and vacancies of various sizes [104-106]. However, defects may relax away [104] or transform into more stable structures [106, 107] over time. For example, highly mobile carbon adatoms appear mostly near vacancies in a metastable structure [104]. Vacancies that migrate near each other are also favored to merge together and reduce the free energy $[105,106]$. The vacancy generating power of electron-beam irradiation has been demonstrated for patterning graphene and cutting out nanoribbons and carbon chains from graphene sheet $[105,108]$. This is done by creating vacancy aggregates with a high-intensity electron beam, and then thinning down the resulting carbon ribbon with a low intensity beam. Thinning is preferred over random electron removal due to the reduced knockout threshold at the edges [109]. The length of the chain can be 
tuned by scanning the beam and the width is controlled by the total dose to the sputtered area. Based on this result, it should be possible to demonstrate arbitrary designs, and serial and parallel patterning, in future work.

Electron-beam irradiation of a graphene-based FETs leads to electron-hole pair generation in the substrate and hole trapping at the graphene- $\mathrm{SiO}_{2}$ interface [6]. This causes a negative shift in the threshold voltage, as well as degradation of device conductivity and carrier mobility. This damage was detectable using Raman spectroscopy at an accumulated dosage as low as 100 electrons $\mathrm{nm}^{-2}$. However, the number of traps appears to saturate after an accumulated dosage of $\sim 1000$ electrons $\mathrm{nm}^{-2}$. The same irradiation causes $\mathrm{hBN}$ to turn amorphous $(\mathrm{aBN})$. The $\mathrm{aBN}$ then either forms clumps or evaporates [98]. Of the two elements in $\mathrm{hBN}$, boron is more likely to be sputtered away by the electron beam due to its lower mass. Once a vacancy forms, however, either element can be sputtered off, and the vacancy grows into a triangular hole [99]. The sputtering behavior of $\mathrm{hBN}$ arises from the small vacancy cross-section of nitrogen atoms in hBN. Additionally, damage is more likely at the edges than inside an hBN flake or sheet [98].

TEM exposure of $\mathrm{MoS}_{2}$ ionizes it via sulfur sputtering, and these vacancies have been observed to collect together into lines [110]. This damage can be prevented by depositing $\mathrm{MoS}_{2}$ layers between graphene sheets. The protective effect of the two graphene sheets under an $80 \mathrm{keV}$ beam was measured as a $\sim 600 \times$ reduction in the vacancy production cross-section [13]. As such, the graphene was able to prevent sputtering from the $\mathrm{MoS}_{2}$. Alternatively, the vacancy formation caused by the focused electron beam in a TEM can be purposefully used to derive metallic MX nanowires from TMDs [111]. However, it remains to be seen whether or not similar useful transformations can be generated with a more conventional electron-beam lithography system.

\section{Nucleon irradiation}

5.1 Protons Proton irradiation is uncommon on Earth, but represents the majority of cosmic radiation incident to the Earth's atmosphere [3]. Studying the effects of proton irradiation on 2DMs can give clues as to their general behavior when irradiated in space environments. For example, protonic bombardment effects on a back-gated $\mathrm{MoS}_{2}$ device leads to trapped holes at the $\mathrm{SiO}_{2}-\mathrm{MoS}_{2}$ interface, which remove electrons from the $\mathrm{MoS}_{2}$ [7]. This increase in the fixed charge degrades the switching behavior of the device, which feeds into a general degradation that worsens with increasing dose. Proton fluences of $10^{12}, 10^{13}$, and $10^{14}$ ions $\mathrm{cm}^{-2}$ were chosen for study. At the lowest fluence, there was a minor reduction in the observed current as a function of drain-source voltage, but no change as a function of gate voltage. On the other hand, there was a $\sim 71 \%$ drop in current with medium irradiation and a $\sim 95 \%$ drop in current with high irradiation at a $5 \mathrm{~V}$ drain bias and a $20 \mathrm{~V}$ gate bias. The devices are able to partially recover after several days, and over very long periods of time they would be expected to recover fully.

5.2 Neutrons Neutron irradiation is a unique result of nuclear reactions. Fast neutrons damage materials mainly through cascading scattering events, and cascade damage to 2DMs will be limited at nanoscale thicknesses. The primary collision from a neutron causes displacement and ionization, and thus we would expect local damage and disorder to be generated. However, to the best of our knowledge, in-depth studies on neutron damage to 2DMs beyond graphene have not been done. These studies tend to focus on bulk graphite, which is used as a neutron moderator in several nuclear reactor designs, and both theoretical [112] and experimental [113] approaches are used to understand radiation damage.

6 Ion irradiation Ion irradiation damage to materials is important due to the degrading defects they can create (see Table 2 for a summary). Controlled ion irradiation can be used to roughly simulate the damage observed in nuclear reactors [124] or induced by cosmic rays. The use of ion beam facilities is motivated by the limited availability of test reactors. Additionally, ion beams are able to damage samples at faster rates than reactors, allowing research on radiation damage to leap ahead of reactor limits. With proper experimental controls, ion beam damage can be used to emulate reactor damage, as has been done for stainless steel [124]. It should be possible to devise a similar means of simulating reactor damage to $2 \mathrm{DMs}$ using ion beam facilities.

There is also interest in using ion irradiation to tailor the properties of $2 \mathrm{D}$ materials by damaging them. Helium ions, due to their small size, are least likely to create defects larger than a single vacancy, and have thus found use as a tool to cleanly cut and shape graphene [116-118]. Helium ion lithography (HIL) and electron beam lithography are comparable in terms of the demonstrated patterning resolution: graphene on $\mathrm{SiO}_{2}$ can be cut into ribbons thinner than $40 \mathrm{~nm}$ [117], while freestanding graphene can be cut into ribbons thinner than $10 \mathrm{~nm} \mathrm{[116].} \mathrm{The} \mathrm{main}$ advantage of HIL over similar techniques is the reduction in the probe beam size, which allows for the creation of finer features [118]. It was noted that the observed dimensions of the patterned features do not always match the intended dimensions. This is due to either a lack or excess of the necessary defects to cause a metal to insulator transition in graphene and section off metallic areas. An optimized dose of helium ions appears necessary to achieve high-resolution nano-scale patterns [117]. This technique can also etch graphene that has already been incorporated into a device [116]. Patterning graphene using a $5 \mathrm{keV}$ xenon atom beam angled at $75^{\circ}$ has also been studied [122]. Vacancy chains are generated in the graphene, caused by energetic ions becoming trapped between the graphene and substrate. Carbon atoms at the edges of the vacancies were found to have bonded to substrate protrusions generated by 
Table 2 Summary of the impacts of ion irradiation on 2DMs.

\begin{tabular}{|c|c|c|c|c|c|}
\hline ion type & material & energy & $\begin{array}{l}\text { fluence range } \\
\text { (ions } \mathrm{cm}^{-2} \text { ) }\end{array}$ & impact & reference \\
\hline proton & graphene & $2 \mathrm{MeV}$ & $10^{15}$ to $6 \times 10^{18}$ & $\begin{array}{l}\text { vacancy and interstitial generation; } \\
\text { surface desorption; increase in } \\
\text { defect density upon suspension of } \\
\text { graphene }\end{array}$ & {$[114,115]$} \\
\hline proton & $\mathrm{MoS}_{2}$ & $10 \mathrm{MeV}$ & $10^{12}$ to $10^{14}$ & $\begin{array}{l}\text { conductivity loss; threshold voltage } \\
\text { shift; interface traps }\end{array}$ & [7] \\
\hline helium & graphene & $35 \mathrm{keV}$ & $10^{16}$ to $10^{17}$ & $\begin{array}{l}\text { graphene etching, cutting, and } \\
\text { patterning }\end{array}$ & [116-118] \\
\hline argon & graphene, $\mathrm{WSe}_{2}$ & $\begin{array}{l}90 \mathrm{eV}, 5 \mathrm{keV} \\
100 \mathrm{keV}\end{array}$ & $10^{11}$ to $10^{15}$ & $\begin{array}{l}\text { isolated defects at lower fluence and } \\
\text { defect coalescence at higher fluence; } \\
\text { creation of scattering centers }\end{array}$ & [119-121] \\
\hline xenon & graphene & $5 \mathrm{keV}$ & $\sim 10^{11}$ to $\sim 10^{14}$ & $\begin{array}{l}\text { vacancy chain generation if angled; } \\
\text { nanomesh generation }\end{array}$ & {$[122]$} \\
\hline swift heavy ion & graphene & $\sim 100 \mathrm{MeV}$ & $\sim 10^{9}$ & $\begin{array}{l}\text { graphene folding, forming of surface } \\
\text { tracks and hillocks }\end{array}$ & [18-20] \\
\hline uranium $\left(\mathrm{U}^{28+}\right)$ & graphene, $\mathrm{MoS}_{2}$ & $1.14 \mathrm{GeV}$ & $4 \times 10^{10}$ to $4 \times 10^{11}$ & $\begin{array}{l}\text { significant changes of structural } \\
\text { properties; conductivity gain at low } \\
\text { fluence and conductivity loss at high } \\
\text { fluence }\end{array}$ & [123] \\
\hline
\end{tabular}

ion irradiation. These protrusions are due to substrate adatoms and can be annealed out at $\sim 750 \mathrm{~K}$, while the vacancies remain until higher temperatures. By annealing above $750 \mathrm{~K}$ and allowing the carbon vacancies to migrate, a graphene nanomesh can be generated [122].

Low-energy angled argon irradiation $\left(90 \mathrm{eV}, 45^{\circ}\right)$ has been used to probe the disordering process of graphene by creating point defects [120]. This bombardment created "activated" A-regions that have in them "structurally disordered" S-regions. Defects remain isolated from each other at doses up to $10^{12}$ ions $\mathrm{cm}^{-2}$, and began to coalesce at $10^{13}$ ions $\mathrm{cm}^{-2}$. At a dose of $10^{14}$ ions $\mathrm{cm}^{-2}$ or greater, the sheet becomes entirely amorphous. As more defects were generated, the A-regions began to overlap and then completely covered the graphene layer. Afterwards, the S-regions increased in number. This process can be observed via Raman spectroscopy as the maximization and then broadening of the D peak. Using the ratio of the $D$ peak to the $G$ peak observed in graphene, this damage can be quantified. However, care must be taken, as the peak hits a maximum once the A-regions fully coalesce. Further damage converts A-regions to S-regions and diminishes the $\mathrm{G}$ peak, lowering this ratio. This effect has been used in a later study to compare the damage done by $100 \mathrm{keV}$ argon ions to the top and bottom layers of bilayer graphene [121]. The substrate can reduce the damage done to graphene by trapping ejected carbon atoms. The interstitial atoms have a small migration barrier, so they will recombine with nearby vacancies.

Damage to $\mathrm{WSe}_{2}$ due to $5 \mathrm{keV}$ argon ions can be probed using scanning tunneling microscopy [119]. The pattern with increasing dose is similar to that of graphene: individual nano-scale impact craters are observed at low doses, and a corrugated surface is created above a dose of $10^{15}$ ions $\mathrm{cm}^{-2}$. While the atoms around the craters are perturbed and restructure themselves, the long-range order is preserved.

Swift heavy ions (SHI) interact with 2DMs mainly through electronic excitations, which also create nanoscale hillocks in the substrate. These protrusions can be used to fold graphene if the beam is angled [19]. Surface tracks can also be formed in the substrate, and they act as acceptors [18]. The relative presence of these two defects is determined by the substrate. For example: $\mathrm{SrTiO}_{3}$ leads to folding and surface tracks; $\mathrm{NaCl}$ leads only to folding; Si(111) leads only to surface tracks [20]. The effect of SHI irradiation from $1.14 \mathrm{GeV}$ uranium ions on graphene and $\mathrm{MoS}_{2}$ FETs depends on the fluence [123]. The lowest fluence $\left(4 \times 10^{10}\right.$ ions $\left.\mathrm{cm}^{-2}\right)$ increased the carrier mobility, while the opposite occurred at higher fluences. The carrier concentration behaved in the opposite manner as the mobility. The lowest and medium fluences $\left(1.5 \times 10^{11}\right)$ reduced the current in the $\mathrm{MoS}_{2}$ device by almost two orders of magnitude, and the highest fluence $\left(4 \times 10^{11}\right)$ destroyed the device. This degradative effect can be seen at all levels of irradiation in both devices, except for the combination of graphene channel and low fluence, perhaps due to some beneficial doping from the substrate. All exposures generated protrusions that could be observed using atomic force microscopy.

7 Single event effects Single event effects (SEEs) are phenomena induced by a single charged particle that deposits energy in a device. Heavy charged particles (protons, alpha particles, ions) are the focus here due to their destructive effects on space-based electronics. These highly 
energetic incident particles can cause sufficient ionization to affect device operation temporarily through a transient, or induce damage leading to failure. Neutron-induced effects are also considered SEEs as neutrons can create charged particle recoils or produce charged particles when captured inside a semiconductor device. Based on the failure mechanism, SEEs can be classified into two categories: hard error (non-recoverable) and soft error (recoverable) [125]. Hard error includes single event latchup [126, 127], single event burnout in power bipolar junction transistors and metal-oxide-semiconductor FETs (MOSFETs) [127, 128], and single event gate rupture causing dielectric breakdown in MOS devices [127, 129]. Soft errors are single event upset and transient, etc. An upset is a state change in caused by radiation-induced charge generation near a sensitive node, while a transient refers to an induced pulse. Transients are an important issue in microelectronic devices such as comparators and operational amplifiers, and can cause latchup in some cases [125]. SEEs are not only important for space; natural terrestrial background radiation and high radiation-field applications also require radiation-hardened devices.

SEEs in traditional microelectronic devices have been well-studied over decades [130]. However, the response of 2DM-based electronics to a single event has not been fully explored. Current studies of ion beam interactions with 2DMs focus on structure and property modification as well as total dose effects. Studies of 2DM-based device susceptibility to SEEs have been very limited, as device and circuit level electronic structures made from 2DMs are still in the research and development stage. Nevertheless, the development of $2 \mathrm{DMs}$ for electronics is promising: a graphene radio frequency receiver integrated circuit (IC) fabricated at IBM Research has been shown to operate at a $4.3 \mathrm{GHz}$ frequency with high performance [131]. Simple ICs based on bilayer $\mathrm{MoS}_{2}$ transistors have also been demonstrated [132]. Therefore, considering the reduced thickness of 2D channel material and the resulting different interaction mechanisms when compared with traditional semiconductor materials, 2DM-based device susceptibility to SEEs could be the basis of a novel and highly relevant future study. Development of radiation hardening technology may be possible based on said future SEE studies.

8 Future outlook The number of materials found to be stable in a 2D configuration appears to be constantly increasing, with silicon [133], silica [134], and titanium trisulfide [135] joining this extended family. Given the pace of progress in this field in recent years, these materials should swiftly find suitable applications matching their properties, or else methods will be developed to tailor them to specific applications. Similarly, the generation and manipulation of individual point defects via particle beams should be expanded to other 2DMs and expand the range of applications for those materials, as well as open up new areas of exploration within the field of materials science and engineering.
More practical challenges to overcome include determining how to achieve very large responsivity for more 2DMs to spur the development of novel photodetectors and solar cells. In general, there is also a dearth of investigation on the effects of high-energy photons and heavy charged particles on 2DMs beyond graphene. Given that the many investigations on graphene have revealed new methods of characterizing and functionalizing this $2 \mathrm{D}$ crystal, similar knowledge and methods should be developed from investigations into other 2DMs to uncover novel physics and develop new electronic devices.

\section{References}

[1] K. S. Novoselov, A. K. Geim, S. V. Morozov et al., Electric field effect in atomically thin carbon films, Science, Science 306(5696), 666-669 (2004).

[2] H. Schmidt, F. Giustiniano, and G. Eda, Electronic transport properties of transition metal dichalcogenide field-effect devices: Surface and interface effects, Chem. Soc. Rev. 44, 7715-7736 (2015).

[3] J. R. Srour and J. M. McGarrity, Radiation effects on microelectronics in space, Proc. IEEE 76(11), 1443-1469 (1988).

[4] H. E. Boesch, F. B. McLean, J. M. McGarrity, and P. S. Winokur, Enhanced flatband voltage recovery in hardened thin MOS capacitors, IEEE Trans. Nucl. Sci. 25(6), 12391245 (1978).

[5] T. R. Oldham, A. J. Lelis, and F. B. McLean, Spatial dependence of trapped holes determined from tunneling analysis and measured annealing, IEEE Trans. Nucl. Sci. 33(6), 1203-1209 (1986).

[6] I. Childres, L. A. Jauregui, M. Foxe et al., Effect of electronbeam irradiation on graphene field effect devices, Appl. Phys. Lett. 97(17), 173109 (2010).

[7] T.-Y. Kim, K. Cho, W. Park et al., Irradiation effects of high-energy proton beams on $\mathrm{MoS}_{2}$ field effect transistors, ACS Nano 8(3), 2774-2781 (2014).

[8] A. Krasheninnikov, P. Lehtinen, A. Foster et al., Embedding transition-metal atoms in graphene: Structure, bonding, and magnetism, Phys. Rev. Lett. 102(12), 126807 (2009).

[9] O. Lehtinen, J. Kotakoski, A. V. Krasheninnikov et al., Effects of ion bombardment on a two-dimensional target: Atomistic simulations of graphene irradiation, Phys. Rev. B 81(15), 153401 (2010).

[10] J. J. Lopez, F. Greer, and J. R. Greer, Enhanced resistance of single-layer graphene to ion bombardment, J. Appl. Phys. 107(10), 104326 (2010).

[11] W. Hu and J. Yang, Defects in phosphorene, J. Phys. Chem. C 119(35), 20474-20480 (2015).

[12] I. S. Kim, V. K. Sangwan, D. Jariwala et al., Influence of stoichiometry on the optical and electrical properties of chemical vapor deposition derived $\mathrm{MoS}_{2}$, ACS Nano 8(10), 10551-10558 (2014).

[13] G. Algara-Siller, S. Kurasch, M. Sedighi et al., The pristine atomic structure of $\mathrm{MoS}_{2}$ monolayer protected from electron radiation damage by graphene, Appl. Phys. Lett. 103, 203107 (2013).

[14] C. Jin, F. Lin, K. Suenaga, and S. Iijima, Fabrication of a freestanding boron nitride single layer and its defect assignments, Phys. Rev. Lett. 102(19), 195505 (2009). 
[15] J.-H. Chen, W. Cullen, C. Jang et al., Defect scattering in graphene, Phys. Rev. Lett. 102(23), 236805 (2009).

[16] H. P. Komsa and A. V. Krasheninnikov, Native defects in bulk and monolayer $\mathrm{MoS}_{2}$ from first principles, Phys. Rev. B 91(12), (2015).

[17] H. Qiu, T. Xu, Z. Wang et al., Hopping transport through defect-induced localized states in molybdenum disulphide, Nature Commun. 4, 2642 (2013).

[18] O. Ochedowski, B. Kleine Bussmann, B. Ban d'Etat et al., Manipulation of the graphene surface potential by ion irradiation, Appl. Phys. Lett. 102(15), 153103 (2013).

[19] S. Akcöltekin, H. Bukowska, T. Peters et al., Unzipping and folding of graphene by swift heavy ions, Appl. Phys. Lett. 98(10), 103103 (2011).

[20] O. Ochedowski, S. Akcöltekin, B. Ban-d'Etat et al., Detecting swift heavy ion irradiation effects with graphene, Nucl. Instrum. Methods Phys. Res. B 314, 18-20 (2013).

[21] H.-P. Komsa, J. Kotakoski, S. Kurasch et al., Two-dimensional transition metal dichalcogenides under electron irradiation: Defect production and doping, Phys. Rev. Lett. 109(3), 035503 (2012).

[22] G. Kresse and J. Furthmüller, Efficient iterative schemes for ab initio total-energy calculations using a plane-wave basis set, Phys. Rev. B 54(16), 11169-11186 (1996).

[23] R. R. Nair, P. Blake, A. N. Grigorenko et al., Fine structure constant defines visual transparency of graphene, Science 320(5881), 1308 (2008).

[24] M. Bernardi, M. Palummo, and J. C. Grossman, Extraordinary sunlight absorption and one nanometer thick photovoltaics using two-dimensional monolayer materials, Nano Lett. 13(8), 3664-3670 (2013).

[25] L. Vicarelli, M. S. Vitiello, D. Coquillat et al., Graphene field-effect transistors as room-temperature terahertz detectors, Nature Mater. 11(10), 865-871 (2012).

[26] F. H. L. Koppens, T. Mueller, P. Avouris et al., Photodetectors based on graphene, other two-dimensional materials and hybrid systems, Nature Nanotechnol. 9(10), 780-793 (2014).

[27] B. Sensale-Rodriguez, R. Yan, M. M. Kelly et al., Broadband graphene terahertz modulators enabled by intraband transitions, Nature Commun. 3, 780 (2012).

[28] W. Knap, M. Dyakonov, D. Coquillat et al., Field effect transistors for terahertz detection: Physics and first imaging applications, Terahertz Waves 30, 1319-1337 (2009).

[29] D. Spirito, D. Coquillat, S. L. De Bonis et al., High performance bilayer-graphene terahertz detectors, Appl. Phys. Lett. 104, 061111 (2014).

[30] X. Cai, A. B. Sushkov, R. J. Suess et al., Sensitive roomtemperature terahertz detection via the photothermoelectric effect in graphene, Nature Nanotechnol. 9(10), 814-819 (2014).

[31] J. R. Piper and S. Fan, Total absorption in a graphene monolayer in the optical regime by critical coupling with a photonic crystal guided resonance, ACS Photon. 1, 347-353 (2014).

[32] M. Freitag, T. Low, and P. Avouris, Increased responsivity of suspended graphene photodetectors, Nano Lett. 13(4), 1644-1648 (2013).

[33] M. Freitag, T. Low, F. N. Xia, and P. Avouris, Photoconductivity of biased graphene, Nature Photon. 7, 53-59 (2013).
[34] B. H. Seo, J. Youn, and M. Shim, Direct laser writing of airstable p-n junctions in graphene, ACS Nano 8, 8831-8836 (2014).

[35] W. H. Wang, H. Y. Nan, Q. Liu et al., Distinct photoresponse in graphene induced by laser irradiation, Appl. Phys. Lett. 106(2), 021121 (2015).

[36] B. Y. Zhang, T. Liu, B. Meng et al., Broadband high photoresponse from pure monolayer graphene photodetector, Nature Commun. 4, 1811 (2013).

[37] Q. Zhang, J. Jie, S. Diao et al., Solution-processed graphene quantum dot deep-UV photodetectors, ACS Nano 9(2), 1561-1570 (2015).

[38] C. Chakraborty, R. Beams, K. M. Goodfellow et al., Optical antenna enhanced graphene photodetector, Appl. Phys. Lett. 105(24), 241114 (2014).

[39] H. Chang, Z. Sun, Q. Yuan et al., Thin film field-effect phototransistors from bandgap-tunable, solution-processed, few-layer reduced graphene oxide films, Adv. Mater. 22(43), 4872-4876 (2010).

[40] B. Chitara, S. B. Krupanidhi, and C. N. R. Rao, Solution processed reduced graphene oxide ultraviolet detector, Appl. Phys. Lett. 99(11), 113114 (2011).

[41] Y. Deng, Z. Luo, N. J. Conrad et al., Black phosphorusmonolayer $\mathrm{MoS}_{2}$ van der Waals heterojunction p-n diode, ACS Nano 8(8), 8292-8299 (2014).

[42] M. Buscema, D. J. Groenendijk, S. I. Blanter et al., Fast and broadband photoresponse of few-layer black phosphorus field-effect transistors, Nano Lett. 14(6), 3347-3352 (2014).

[43] Y. Deng, N. J. Conrad, Z. Luo et al., Towards highperformance two-dimensional black phosphorus optoelectronic devices: The role of metal contacts, 2014 IEEE Int. Electron Devices Meeting, 5.2.1-5.2.4 (2014).

[44] J. Wu, G. K. W. Koon, D. Xiang et al., Colossal ultraviolet photoresponsivity of few-layer black phosphorus, ACS Nano 9(8), 8070-8077 (2015).

[45] P. Hu, L. Wang, M. Yoon et al., Highly responsive ultrathin $\mathrm{GaS}$ nanosheet photodetectors on rigid and flexible substrates, Nano Lett. 13(4), 1649-1654 (2013).

[46] P. Hu, Z. Wen, L. Wang et al., Synthesis of few-layer GaSe nanosheets for high performance photodetectors, ACS Nano 6(7), 5988-5994 (2012).

[47] S. Lei, L. Ge, Z. Liu et al., Synthesis and photoresponse of large GaSe atomic layers, Nano Lett. 13(6), 2777-2781 (2013).

[48] F. Liu, H. Shimotani, H. Shang et al., High-sensitivity photodetectors based on multilayer GaTe flakes, ACS Nano 8(1), 752-760 (2014).

[49] X. Yuan, L. Tang, P. Wang et al., Wafer-scale arrayed p-n junctions based on few-layer epitaxial GaTe, Nano Res. 8, 3332-3341 (2014).

[50] Z. Wang, M. Safdar, M. Mirza et al., High-performance flexible photodetectors based on GaTe nanosheets, Nanoscale 7(16), 7252-7258 (2015).

[51] R. B. Jacobs-Gedrim, M. Shanmugam, N. Jain et al., Extraordinary photoresponse in two-dimensional $\operatorname{In}(2)$ Se(3) nanosheets, ACS Nano 8(1), 514-521 (2014).

[52] Q.-L. Li, C.-H. Liu, Y.-T. Nie et al., Phototransistor based on single $\mathrm{In}_{2} \mathrm{Se}_{3}$ nanosheets, Nanoscale 6, 14538-14542 (2014).

[53] Z. Chen, J. Biscaras, and A. Shukla, A high performance graphene/few-layer InSe photo-detector, Nanoscale 7(14), 5981-5986 (2015). 
[54] S. R. Tamalampudi, Y.-Y. Lu, U. R. Kumar et al., High performance and bendable few-layered InSe photodetectors with broad spectral response, Nano Lett. 14(5), 2800-2806 (2014).

[55] W.Zheng, T. Xie, Y. Zhou et al., Patterning two-dimensional chalcogenide crystals of $\mathrm{Bi}_{2} \mathrm{Se}_{3}$ and $\mathrm{In}_{2} \mathrm{Se}_{3}$ and efficient photodetectors, Nature Commun. 6, 6972 (2015).

[56] W. Zhang, J.-K. Huang, C.-H. Chen et al., High-gain phototransistors based on a CVD $\mathrm{MoS}_{2}$ monolayer, Adv. Mater. 25(25), 3456-3461 (2013).

[57] W. Zhang, C.-P. Chuu, J.-K. Huang et al., Ultrahigh-gain photodetectors based on atomically thin graphene- $\mathrm{MoS}_{2}$ heterostructures, Sci. Rep. 4, 3826 (2014).

[58] Z. Yin, H. Li, H. Li et al., Single-layer $\mathrm{MoS}_{2}$ phototransistors, ACS Nano 6(1), 74-80 (2012).

[59] O. Lopez-Sanchez, D. Lembke, M. Kayci et al., Ultrasensitive photodetectors based on monolayer $\mathrm{MoS}_{2}$, Nature Nanotechnol. 8(7), 497-501 (2013).

[60] W. Choi, M. Y. Cho, A. Konar et al., High-detectivity multilayer $\operatorname{MoS}(2)$ phototransistors with spectral response from ultraviolet to infrared, Adv. Mater. 24(43), 5832-5836 (2012).

[61] S. H. Yu, Y. Lee, S. K. Jang et al., Dye-sensitized $\mathrm{MoS}_{2}$ photodetector with enhanced spectral photoresponse, ACS Nano 8(8), 8285-8291 (2014).

[62] H.-J. Kwon, J. Jang, S. Kim et al., Electrical characteristics of multilayer $\mathrm{MoS}_{2}$ transistors at real operating temperatures with different ambient conditions, Appl. Phys. Lett. 105(15), 152105 (2014).

[63] J. Kwon, Y. K. Hong, G. Han et al., Phototransistors: Giant photoamplification in indirect-bandgap multilayer $\mathrm{MoS}_{2}$ phototransistors with local bottom-gate structures, Adv. Mater. 27(13), 2126-2126 (2015).

[64] D.-H. Kang, M.-S. Kim, J. Shim et al., High-performance transition metal dichalcogenide photodetectors enhanced by self-assembled monolayer doping, Adv. Funct. Mater. 25(27), 4219-4227 (2015).

[65] X. Wang, P. Wang, J. Wang et al., Ultrasensitive and broadband $\mathrm{MoS}_{2}$ photodetector driven by ferroelectrics, Adv. Mater. 27(42), 6575-6581 (2015).

[66] E. Zhang, Y. Jin, X. Yuan et al., $\mathrm{ReS}_{2}$-based field-effect transistors and photodetectors, Adv. Funct. Mater. 25, 4076-4082 (2015).

[67] N. Perea-López, A. L. Elías, A. Berkdemir et al., Photosensor device based on few-layered $\mathrm{WS}_{2}$ films, Adv. Funct. Mater. 23(44), 5511-5517 (2013).

[68] B. W. H. Baugher, H. O. H. Churchill, Y. Yang, and P. Jarillo-Herrero, Optoelectronic devices based on electrically tunable $\mathrm{p}-\mathrm{n}$ diodes in a monolayer dichalcogenide, Nature Nanotechnol. 9(4), 262-267 (2014).

[69] A. Pospischil, M. M. Furchi, and T. Mueller, Solar-energy conversion and light emission in an atomic monolayer $\mathrm{p}-\mathrm{n}$ diode, Nature Nanotechnol. 9(4), 257-261 (2014).

[70] C. Lan, C. Li, Y. Yin, and Y. Liu, Large-area synthesis of monolayer $\mathrm{WS}_{2}$ and its ambient-sensitive photodetecting performance, Nanoscale 7(14), 5974-5980 (2015).

[71] Y. Huang, E. Sutter, J. T. Sadowski et al., Tin disulfide-an emerging layered metal dichalcogenide semiconductor: Materials properties and device characteristics, ACS Nano 8(10), 10743-10755 (2014).
[72] Y. Huang, H.-X. Deng, K. Xu et al., Highly sensitive and fast phototransistor based on large size CVD-grown $\mathrm{SnS}_{2}$ nanosheets, Nanoscale 7(33), 14093-14099 (2015).

[73] G. Su, V. G. Hadjiev, P. E. Loya et al., Chemical vapor deposition of thin crystals of layered semiconductor $\mathrm{SnS}_{2}$ for fast photodetection application, Nano Lett. 15(1), 506-513 (2015).

[74] J. Yao, J. Shao, Y. Wang et al., Ultra-broadband and high response of the $\mathrm{Bi}_{2} \mathrm{Te}_{3}-\mathrm{Si}$ heterojunction and its application as a photodetector at room temperature in harsh working environments, Nanoscale 7(29), 12535-12541 (2015).

[75] J. Aumanen, A. Johansson, J. Koivistoinen et al., Patterning and tuning of electrical and optical properties of graphene by laser induced two-photon oxidation, Nanoscale 7(7), 2851-2855 (2015).

[76] K. Roy, M. Padmanabhan, S. Goswami et al., Graphene$\mathrm{MoS}_{2}$ hybrid structures for multifunctional photoresponsive memory devices, Nature Nanotechnol. 8, 826-830 (2013).

[77] F. Liu and S. Kar, Quantum carrier reinvestment-induced ultrahigh and broadband photocurrent responses in graphene-silicon junctions, ACS Nano 8(10), 10270-10279 (2014).

[78] X. An, F. Liu, Y. J. Jung, and S. Kar, Tunable graphenesilicon heterojunctions for ultrasensitive photodetection, Nano Lett. 13, 909-916 (2013).

[79] B. K. Sarker, I. Childres, E. Cazalas et al., Gate-tunable and high responsivity graphene phototransistors on undoped semiconductor substrates arXiv:1409.5725 (2014).

[80] G. Konstantatos, M. Badioli, L. Gaudreau et al., Hybrid graphene-quantum dot phototransistors with ultrahigh gain, Nature Nanotechnol. 7, 363-368 (2012).

[81] D. H. Shin, S. Kim, J. M. Kim et al., Graphene/Si-quantumdot heterojunction diodes showing high photosensitivity compatible with quantum confinement effect, Adv. Mater. 27, 2614-2620 (2015).

[82] H. Qiao, J. Yuan, Z. Xu et al., Broadband photodetectors based on graphene- $\mathrm{Bi}_{2} \mathrm{Te}_{3}$ heterostructure, ACS Nano 9(2), 1886-1894 (2015).

[83] V. Patil, A. Capone, S. Strauf, and E.-H. Yang, Improved photoresponse with enhanced photoelectric contribution in fully suspended graphene photodetectors, Sci. Rep. 3, 2791 (2013).

[84] H. S. Lee, S.-W. Min, Y.-G. Chang et al., $\mathrm{MoS}_{2}$ nanosheet phototransistors with thickness-modulated optical energy gap, Nano Lett. 12(7), 3695-3700 (2012).

[85] M. Buscema, M. Barkelid, V. Zwiller et al., Large and tunable photothermoelectric effect in single-layer $\mathrm{MoS}_{2}$, Nano Lett. 13(2), 358-363 (2013).

[86] Y. Son, Q. H. Wang, J. A. Paulson et al., Layer number dependence of $\mathrm{MoS}_{2}$ photoconductivity using photocurrent spectral atomic force microscopic imaging, ACS Nano 9(3), 2843-2855 (2015).

[87] Z. Wang, K. Xu, Y. Li et al., Role of Ga vacancy on a multilayer GaTe phototransistor, ACS Nano 8(5), 4859-4865 (2014).

[88] T. N. Lin, K. H. Chih, C. T. Yuan et al., Laser-ablation production of graphene oxide nanostructures: From ribbons to quantum dots, Nanoscale 7(6), 2708-2715 (2015).

[89] M. Foxe, G. Lopez, I. Childres et al., Detection of ionizing radiation using graphene field effect transistors, 2009 IEEE Nucl. Sci. Symp. Conf. Rec. 90-95 (2009). 
[90] A. Patil, O. Koybasi, G. Lopez et al., Graphene field effect transistor as radiation sensor, IEEE Nucl. Sci. Symp. Conf. Rec. 455-459 (2011).

[91] M.-X. Han, Z.-Y. Ji, L.-W. Shang et al., $\gamma$ radiation caused graphene defects and increased carrier density, Chin. Phys. B 20(8), 086102 (2011).

[92] G. R. Bhimanapati, M. Wetherington, S. Mahabir, and J. A. Robinson, Synthesis and radiation response of BCON: A graphene oxide and hexagonal boron nitride hybrid, 2D Mater. 3(2), 025028 (2016).

[93] H. Hong, Y. Zhang, J. W. Engle et al., In vivo targeting and positron emission tomography imaging of tumor vasculature with Ga-66-labeled nano-graphene, Biomaterials 33(16), 4147-4156 (2012).

[94] Y. Wang, Y. Feng, F. Mo et al., Influence of irradiation upon few-layered graphene using electron-beams and gammarays, Appl. Phys. Lett. 105(2), 023102 (2014).

[95] B. Zhang, L. Li, Z. Wang et al., Radiation induced reduction: An effective and clean route to synthesize functionalized graphene, J. Mater. Chem. 22(16), 7775 (2012).

[96] Y. Zhang, H.-L. Ma, Q. Zhang et al., Facile synthesis of well-dispersed graphene by $\gamma$-ray induced reduction of graphene oxide, J. Mater. Chem. 22(26), 13064 (2012).

[97] R. F. Egerton, P. Li, and M. Malac, Radiation damage in the TEM and SEM, Micron 35(6), 399-409 (2004).

[98] J. S. Kim, K. B. Borisenko, V. Nicolosi, and A. I. Kirkland, Controlled radiation damage and edge structures in boron nitride membranes, ACS Nano 5(5), 3977-3986 (2011).

[99] J. C. Meyer, A. Chuvilin, G. Algara-Siller et al., Selective sputtering and atomic resolution imaging of atomically thin boron nitride membranes, Nano Lett. 9(7), 2683-2689 (2009).

[100] J. Kotakoski, C. H. Jin, O. Lehtinen et al., Electron knockon damage in hexagonal boron nitride monolayers, Phys. Rev. B 82(11), 113404 (2010).

[101] R. Zan, Q. M. Ramasse, R. Jalil et al., Control of radiation damage in $\mathrm{MoS}_{2}$ by graphene encapsulation, ACS Nano 7(11), 10167-10174 (2013).

[102] J. Kotakoski, D. Santos-Cottin, and A. V. Krasheninnikov, Stability of graphene edges under electron beam: Equilibrium energetics versus dynamic effects, ACS Nano 6(1), 671-676 (2012).

[103] X. Wei, D.-M. Tang, Q. Chen et al., Local Coulomb explosion of boron nitride nanotubes under electron beam irradiation, ACS Nano 7(4), 3491-3497 (2013).

[104] A. Hashimoto, K. Suenaga, A. Gloter et al., Direct evidence for atomic defects in graphene layers, Nature 430 (7002), 870-873 (2004).

[105] A. W. Robertson and J. H. Warner, Atomic resolution imaging of graphene by transmission electron microscopy, Nanoscale 5(10), 4079-4093 (2013).

[106] S. T. Skowron, I. V Lebedeva, A. M. Popov, and E. Bichoutskaia, Energetics of atomic scale structure changes in graphene, Chem. Soc. Rev. 44(10), 3143-3176 (2015).

[107] J. H. Warner, M. H. Rümmeli, L. Ge et al., Structural transformations in graphene studied with high spatial and temporal resolution, Nature Nanotechnol. 4(8), 500-504 (2009).

[108] A. Chuvilin, J. C. Meyer, G. Algara-Siller, and U. Kaiser, From graphene constrictions to single carbon chains, New J. Phys. 11, 1-10 (2009).

[109] C. Jin, H. Lan, L. Peng et al., Deriving carbon atomic chains from graphene, Phys. Rev. Lett. 102(20), 205501 (2009).
[110] S. Wang, G.-D. Lee, S. Lee et al., Detailed atomic reconstruction of extended line defects in monolayer $\mathrm{MoS}_{2}$, ACS Nano 10, 5419-5430 (2016). acsnano.6b01673

[111] J. Lin, O. Cretu, W. Zhou et al., Flexible metallic nanowires with self-adaptive contacts to semiconducting transitionmetal dichalcogenide monolayers, Nature Nanotechnol. 9(6), 436-442 (2014).

[112] H. J. Christie, M. Robinson, D. L. Roach et al., Simulating radiation damage cascades in graphite, Carbon 81(1), 105-114 (2015).

[113] J. Eapen, R. Krishna, T. D. Burchell, and K. L. Murty, Early damage mechanisms in nuclear grade graphite under irradiation, Mater. Res. Lett. 2(1), 43-50 (2013).

[114] S. Mathew, T. K. Chan, D. Zhan et al., The effect of layer number and substrate on the stability of graphene under $\mathrm{MeV}$ proton beam irradiation, Carbon 49(5), 1720-1726 (2011).

[115] S. Mathew, T. K. Chan, D. Zhan et al., Mega-electron-volt proton irradiation on supported and suspended graphene: A Raman spectroscopic layer dependent study, J. Appl. Phys. 110(8), 084309 (2011).

[116] M. C. Lemme, D. C. Bell, J. R. Williams et al., Etching of graphene devices with a helium ion beam, ACS Nano 3(9), 2674-2676 (2009).

[117] Y. Naitou, T. Iijima, and S. Ogawa, Direct nano-patterning of graphene with helium ion beams, Appl. Phys. Lett. 106(3), 033103 (2015).

[118] D. C. Bell, M. C. Lemme, L. A. Stern et al., Precision cutting and patterning of graphene with helium ions, Nanotechnology 20(45), 455301 (2009).

[119] T. Schimmel, H. Fuchs, R. Sander, and M. Lux-Steiner, Atomically resolved STM imaging of ion-bombarded $\mathrm{WSe}_{2}$, Ultramicroscopy 42-44(Part 1), 683-688 (1992).

[120] M. M. Lucchese, F. Stavale, E. H. M. Ferreira et al., Quantifying ion-induced defects and Raman relaxation length in graphene, Carbon 48(5), 1592-1597 (2010).

[121] M. Kalbac, O. Lehtinen, A. V. Krasheninnikov, and J. Keinonen, Ion-irradiation-induced defects in isotopicallylabeled two layered graphene: Enhanced in-situ annealing of the damage, Adv. Mater. 25(7), 1004-1009 (2013).

[122] S. Standop, O. Lehtinen, C. Herbig et al., Ion impacts on graphene/Ir(111): Interface channeling, vacancy funnels, and a nanomesh, Nano Lett. 13(5), 1948-1955 (2013).

[123] O. Ochedowski, K. Marinov, G. Wilbs et al., Radiation hardness of graphene and $\mathrm{MoS}_{2}$ field effect devices against swift heavy ion irradiation, J. Appl. Phys. 113(21), 214306 (2013).

[124] G. S. Was, Z. Jiao, E. Getto et al., Emulation of reactor irradiation damage using ion beams, Scr. Mater. 88, 33-36 (2014).

[125] M. Nicolaidis, Soft Errors In Modern Electronic Systems (Springer Science + Business Media LLC, New York, 2011).

[126] W. A. Kolasinski, J. B. Blake, J. K. Anthony et al., Simulation of cosmic-ray induced soft errors and latchup in integrated-circuit computer memories, IEEE Trans. Nucl. Sci. 26(6), 5087-5091 (1979).

[127] F. W. Sexton, Destructive single-event effects in semiconductor devices and ICs, IEEE Trans. Nucl. Sci. 50(3), 603-621 (2003).

[128] A. E. Waskiewicz, J. W. Groninger, V. H. Strahan, and D. M. Long, Burnout of power MOS transistors with heavy ions of Californium-252, IEEE Trans. Nucl. Sci. 33(6), 1710-1713 (1986). 
[129] J. C. Pickel and J. T. Blandford, Cosmic-ray-induced errors in MOS devices, IEEE Trans. Nucl. Sci. 27(2), 1006-1015 (1980).

[130] P. E. Dodd and L. W. Massengill, Basic mechanisms and modeling of single-event upset in digital microelectronics, IEEE Trans. Nucl. Sci. 50(3), 583-602 (2003).

[131] S.-J. Han, A. V. Garcia, S. Oida et al., Graphene radio frequency receiver integrated circuit, Nature Commun. 5, 3086 (2014).

[132] H. Wang, L. Yu, Y.-H. Lee et al., Integrated circuits based on bilayer $\mathrm{MoS}_{2}$ transistors, Nano Lett. 12(9), 4674-4680 (2012).
[133] A. Kara, H. Enriquez, A. P. Seitsonen et al., A review on silicene - New candidate for electronics, Surf. Sci. Rep. 67(1), 1-18 (2012).

[134] P. Y. Huang, S. Kurasch, A. Srivastava et al., Direct imaging of a two-dimensional silica glass on graphene, Nano Lett. 12(2), 1081-1086 (2012).

[135] A. Lipatov, P. Wilson, M. Shekhirev et al., Few-layer titanium trisulfide (TiS3) field-effect transistors, Nanoscale 7, 12291-12296 (2015).

[136] K. S. Novoselov, V. I. Fal'ko, L. Colombo et al., A roadmap for graphene, Nature 490(7419), 192-200 (2012). 